\title{
Discretization-dependent model for weakly connected excitable media
}

\author{
Pedro André Arroyo ${ }^{1,2}$, Sergio Alonso ${ }^{1,2},{ }^{*}$ and Rodrigo Weber dos Santos ${ }^{1 \dagger}$ \\ ${ }^{1}$ Graduate Program in Computational Modeling, \\ Universidade Federal de Juiz de Fora, Juiz de Fora, Brazil \\ ${ }^{2}$ Department of Physics, \\ Universitat Politècnica de Catalunya, Barcelona, Spain
}

(Dated: April 16, 2018)

\begin{abstract}
Pattern formation has been widely observed in extended chemical and biological processes. Although the biochemical systems are highly heterogeneous, homogenized continuum approaches formed by partial differential equations have been employed frequently. Such approaches are usually justified by the difference of scales between the heterogeneities and the characteristic spatial size of the patterns. Under different conditions, for example under weak coupling, discrete models are more adequate. However, discrete models may be less manageable, for instance, in terms of numerical implementation and mesh generation, than the associated continuum models. Here we study a model to approach discreteness which permits the computer implementation on general unstructured meshes. The model is cast as a partial differential equation but with a parameter that depends not only on heterogeneities sizes, as in the case of quasi-continuum models, but also on the discretization mesh. Therefore, we refer to it as a discretization-dependent model. We validate the approach in a generic excitable media that simulates three different phenomena: the propagation of action membrane potential in cardiac tissue; in myelinated axons of neurons; and concentration waves in chemical microemulsions.
\end{abstract}

PACS numbers: May be entered using the \pacs\{\#1\} command.

\section{INTRODUCTION}

Chemical and biochemical reactions outside of the thermodynamic equilibrium are highly nonlinear. In continuum media and in combination with diffusive transport such nonlinear reactions can produce the formation of spatio-temporal patterns [1]. A particular example of pattern formation is traveling waves in excitable media [2]. Such dynamics has been extensively studied [3], [4] due to its relation with important physiological processes like heart beating [5] and stimulus propagation in neuronal networks [6]. Excitable waves have been also observed in diverse chemical systems like the BelousovZhabotinsky reaction [7] and CO oxidation in catalytic surfaces [8].

For the aforementioned models, homogenized continuum approaches formed by partial differential equations (PDEs) have been extensively employed. Homogenization techniques allow continuum models to capture the discrete and small scale details of the particular system or phenomena in terms of reaction-diffusion equations:

$$
\partial_{t} u^{j}=F^{j}(\boldsymbol{u})+\nabla \cdot\left(\sigma \nabla u^{j}\right),
$$

where $\boldsymbol{u}$ is the vector of variables of interest, $u^{j}$, and $\sigma$ is the homogenized or effective diffusion coefficient. This is the case of cardiac tissue, formed by a discrete grid of cardiac cells, the brain, formed by a complex discrete network of neurons, and some chemical reactions that takes place inside droplets that form a set of

\footnotetext{
*s.alonso@upc.edu

† rodrigo.weber@ufjf.edu.br
}

weakly connected discrete microscopic reactors [9]. The corresponding models of such discrete systems have been transformed into homogenized continuum versions [10] with effective parameters [11]. For instance, the differences among nonlinear reactions can be treated with a mean-field approach and the heterogeneous diffusion can be homogenized via the classical Bruggeman's approximation of effective medium [12].

However, such homogenized continuum descriptions may fail when characteristic lengths of patterns are small and close to the scale of the details of the system. Under such conditions a detailed and microscopic description is typically employed, usually based on heterogeneous partial differential equations. Another possibility is the use of discrete models with effective parameters mimicking the homogenization performed for continuum models. Here, a discrete model refers to a discrete set of equations,

$$
\frac{d u_{i}^{j}}{d t}=F^{j}\left(\boldsymbol{u}_{i}\right)+\sum_{k} G^{j}\left(u_{k}^{j}\right),
$$

where $i$ identifies one specific discrete component of the system (e.g. a cell or a droplet), $\boldsymbol{u}$ is the vector of variables of interest, $u^{j}$, and the summation over $k$ highlights that the components are not independent from each other. In the simple case of $1 \mathrm{D}$ model this summation may involve only first neighbors (to represent, for instance, the discrete Laplacian operator). Nevertheless, it is possible to use complex networks in this summation term $[6,13]$. In summary, the continuum space is replaced by a discrete set of components whereas time is still continuous, leading us to a system of coupled ordinary differential equations. 
For instance, for the case of action potential propagation on cardiac tissue, different microscopic models based on heterogeneous PDEs [14-18] and on discrete models [18-20] were proposed to investigate important aspects of cardiac diseases.

Unfortunately, both alternatives pose non-trivial computational challenges. The multi-scale nature of complex phenomena leads to a large number of equations when microscopic or heterogeneous continuum models are adopted. On the other hand, discrete models demand precise representation and location of each individual component of the system, a property that challenges mesh generation. Ideally, we would like to have a model that: 1) captures the complex features of the phenomena, i.e. behave as well as the microscopic or discrete models; 2 ) is computationally less expensive than the microscopic model; and 3) is able to use general unstructured meshes as those routinely generated for the solution of PDEs.

We have recently proposed a new model [21] that, for the particular case of slow propagation on cardiac tissue, was able to fulfill all the above requirements. This model can be seen as an extension of the works on quasicontinuum models [22-25] that propose partial differential equations (PDEs) that approach discreteness. Such quasi-continuum models take into account higher order terms for the diffusion operator together with the characteristic length of the system to approach, in a continuum way, the underlying discrete nature of the problem, see $[26]$ for a review. It is worth noting that similar models were also proposed recently in the field of phase transitions [27] and in structural mechanics [28, 29].

Classical homogenization techniques and quasicontinuum models generate PDEs by upscaling some information of the microscopic/discrete structure of the system, $\mathfrak{L}$. This information is usually embedded in some coefficients of the generated PDEs. For instance, the coefficient $\sigma$ in eq.(1) would depend on the microscopic features: $\sigma(\mathfrak{L})$. The main idea of our model is to use also some information of the mesh or discretization, $\mathfrak{M}$. Therefore, the coefficients of the PDEs depend on both $\mathfrak{L}$ and $\mathfrak{M}$, e.g., we would have $\sigma(\mathfrak{L}, \mathfrak{M})$ in eq.(1). Thus, we call our new model a Discretization Dependent Model $(D D M)$.

In this work, we continue previous investigations [21] of DDM by evaluating its performance for the description of three different excitable media, cardiac tissue with reduced conductivity, slow impulse conduction in myelinated axons, and chemical reaction inside small droplets surrounded by oil. We chose these phenomena since each one of them presents very distinct scales of spatiotemporal dynamics. Furthermore, we show that our new proposed $D D M$ model can behave as a quasi-continuum model, when the discretization size of the mesh goes to zero, and as a pure discrete model, when the mesh $\mathfrak{M}$ matches the topology of the discrete system, $\mathfrak{L}$.

Our results suggest that our new $D D M$, for all the three problems, outperforms both the classical homogenized continuum model as well the quasi-continuum models. In addition, its performance is similar to the discrete model, but with the advantage of its flexibility in terms of numerical mesh that does not need to precisely match the topology of the discrete system.

\section{MODELS}

We consider here a set of two reaction-diffusion equations with reaction terms described by the modified Fitzhugh-Nagumo model [30]. Therefore, we take eq.(1) for two components, $j=2$, to obtain the next system of equations:

$$
\begin{aligned}
\partial_{t} u & =f(u, v)+\partial_{x}\left(\sigma \partial_{x} u\right), \\
\partial_{t} v & =g(u, v)+\delta \partial_{x}\left(\sigma \partial_{x} v\right),
\end{aligned}
$$

where the variables $u$ and $v$ may correspond to chemical concentrations or action membrane potential depending on the particular application, and $f$ and $g$ are, in general, non-linear functions. Here we consider:

$$
\begin{aligned}
f(u, v) & =k[u(1-u)(u-a)-u v] \\
g(u, v) & =k[\varepsilon(b u-v)] .
\end{aligned}
$$

The model is based on the Fitzhugh-Nagumo model $[31,32]$ where the term - uv prevents negative values of the variable $u$. It is a generic model of excitable medium and after a proper initial condition it produces the formation of traveling excitation waves in a 1D system, see fig.1, or spiral and scroll waves in higher dimensions. The corresponding boundary conditions and initials values are:

$$
\begin{aligned}
\partial_{x} u(x, t) & =\partial_{x} v(x, t)=0, \quad \forall x \in \partial \Omega, \\
u(x, 0) & =u_{0}, v(x, 0)=v_{0} \quad x \in \Omega_{0} \subset \Omega .
\end{aligned}
$$

Next we show different mathematical models that can be used to describe an heterogeneous reaction-diffusion system, going from a detailed description to homogenized approximations of the system.

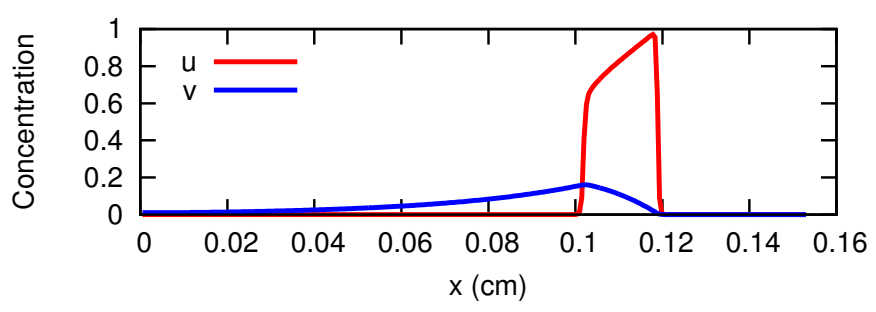

FIG. 1. Snapshot of an excitable traveling wave obtained from a numerical simulation using FitzHugh-Nagumo model as reaction term, see eqs.(3-6). 


\section{A. Previous models}

First, we perform a systematic study of the different models employed during the last years in the modeling of heterogeneous reaction-diffusion systems. Below, in Fig.2 there is comparison among all the approaches under two different conditions.

\section{Heterogeneous multi-scale model}

The most exact description corresponds to the heterogeneous general case which is given by the next set of reaction-diffusion equations for two generic variables $u$ and $v$ with spatially dependent coefficients:

$$
\begin{aligned}
& \mathcal{X}(x) \partial_{t} u=\theta(x) f(u, v)+\partial_{x}\left(\sigma(x) \partial_{x} u\right) \\
& \mathcal{X}(x) \partial_{t} v=\theta(x) g(u, v)+\delta \partial_{x}\left(\sigma(x) \partial_{x} v\right) .
\end{aligned}
$$

$\sigma(x)$ is the spatially-dependent conductivity or diffusion coefficient. Function $\theta(x)$ identifies the spatial location where the reactions appear, see more details below in Section IV. Function $\mathcal{X}(x)$ depends on the particular case considered, see Table I. Parameter $\delta$ is the ratio of diffusion coefficients for $u$ and $v$.

We named this model Heterogeneous Multi-scale Model (HMM), and although it is the best approach to the real case, it is rarely used, because of its high computational costs.

For the excitable dynamics induced by eqs. $(5,6)$, a wave propagates through an one dimensional system. See two examples in Fig.2 for two different values of conductivities. For both cases we have $\mathcal{X}=\theta=1$ and $\delta=0$. The reaction terms are given by eqs.(3-6). The conductivity $\sigma(x)$ is periodic on space with a high value of $\sigma_{0}$ for the length $l_{0}=100 \mu \mathrm{m}$ that alternates to a low value of $\sigma_{1}$ for the length $l_{1}=0.5 \mu \mathrm{m}$. For normal conduction we use $\sigma_{0}=1.035 \times 10^{-3}$ and $\sigma_{1}=1.035 \times 10^{-5} \mu \mathrm{m}^{2} / \mathrm{ms}$; for weak conduction we decrease the second parameter, $\sigma_{1}=2.96 \times 10^{-7} \mu \mathrm{m}^{2} / \mathrm{ms}$.

\section{Homogenized continuum model}

If the spatial changes in the functions $\sigma(x), \theta(x)$ and $\mathcal{X}(x)$ are smaller than any characteristic length of the obtained spatial patterns, the $H M M$ can be reduced to a homogenized Continuum Model (CM). These functions are averaged to particular homogeneous values $\bar{\sigma}, \bar{\theta}, \overline{\mathcal{X}}$ by upscaling some important information, $\mathfrak{L}$, from the detailed heterogeneous system to arrive at:

$$
\begin{aligned}
\overline{\mathcal{X}} \partial_{t} u & =\bar{\theta} f(u, v)+\bar{\sigma} \partial_{x}^{2} u, \\
\overline{\mathcal{X}} \partial_{t} v & =\bar{\theta} g(u, v)+\delta \bar{\sigma} \partial_{x}^{2} v .
\end{aligned}
$$

which forms a set of homogeneous partial differential equations.
In Fig. 2 for the Homogenized Model all the parameters are the same as those used for the HMM. The homogenized conductivity is calculated by $\bar{\sigma}=\left(\ell_{0}+\right.$ $\left.\ell_{1}\right) /\left(\ell_{0} / \sigma_{0}+\ell_{1} / \sigma_{1}\right)$.

In Fig. 2(A,B) we can observe that under normal conditions, the $C M$ approximates well the dynamics of the $H M M$. However, for weak conduction, the $C M$ fails to reproduce the slow dynamics (see Fig.2(C-D)).

\section{Homogenized discrete model}

When the continuum approach fails, an alternative is to use a homogenized Discrete Model (DM) with a characteristic length $\ell$ that comes from the underlying inhomogeneity of the system, i.e., it also comes from $\mathfrak{L}$, mimicking, for example, the length of a single cell.

The dynamics of a single element of such discrete system follows the same non-linear reactions and the interaction with the two first neighbors is given by the discrete Laplacian $\partial_{\ell}^{2}$ :

$$
\begin{aligned}
\overline{\mathcal{X}} \partial_{t} u_{i} & =\bar{\theta} f\left(u_{i}, v_{i}\right)+\bar{\sigma} \partial_{\ell}^{2} u_{i}, \\
\overline{\mathcal{X}} \partial_{t} v_{i} & =\bar{\theta} g\left(u_{i}, v_{i}\right)+\delta \bar{\sigma} \partial_{\ell}^{2} v_{i},
\end{aligned}
$$

where $\partial_{\ell}^{2} u_{i}=\frac{u_{i+1}-2 u_{i}+u_{i-1}}{\ell^{2}}, \bar{\sigma}, \overline{\mathcal{X}}, \bar{\theta}$ are the same homogenized values of the functions $\sigma(x), \theta(x)$ and $\mathcal{X}(x)$ as in eqs. $(9,10)$.

The computational integration of these equations is fast and its solutions approximate very well those of the $H M M$, under both normal and weak conduction, as shown in Fig.2. However, as mentioned before, the downside is that the length $\ell$ is fixed, and the model can not be solved on a arbitrary mesh.

\section{Quasi-continuum model}

To overcome the above mentioned problem, continuum models have been proposed in the past with the main goal of approximating discreteness. These models take into account the discrete nature of the phenomena, i.e., the characteristic length $\ell$, together with high order approximations, obtained for instance via Padé series [25]. Such continuum approximations are usually called quasicontinuum models QCM [22-25]. Applying these techniques to our equations would give us:

$$
\begin{aligned}
& \overline{\mathcal{X}} \partial_{t} u=\bar{\theta} f(u, v)+\left(\frac{\bar{\sigma}}{1-\kappa_{1} \partial_{x}^{2}}\right) \partial_{x}^{2} u \\
& \overline{\mathcal{X}} \partial_{t} v=\bar{\theta} g(u, v)+\left(\frac{\delta \bar{\sigma}}{1-\kappa_{1} \partial_{x}^{2}}\right) \partial_{x}^{2} v,
\end{aligned}
$$

where $\kappa_{1}=\frac{\ell^{2}}{12}$.

Fig.2 shows that $Q C M$ indeed outperforms $C M$ and keeps the desired feature of a classical PDE, i.e., can be solved on any arbitrary mesh. Nevertheless, for the case of weak coupling, the solution is still far from our gold standard, the $H M M$. 

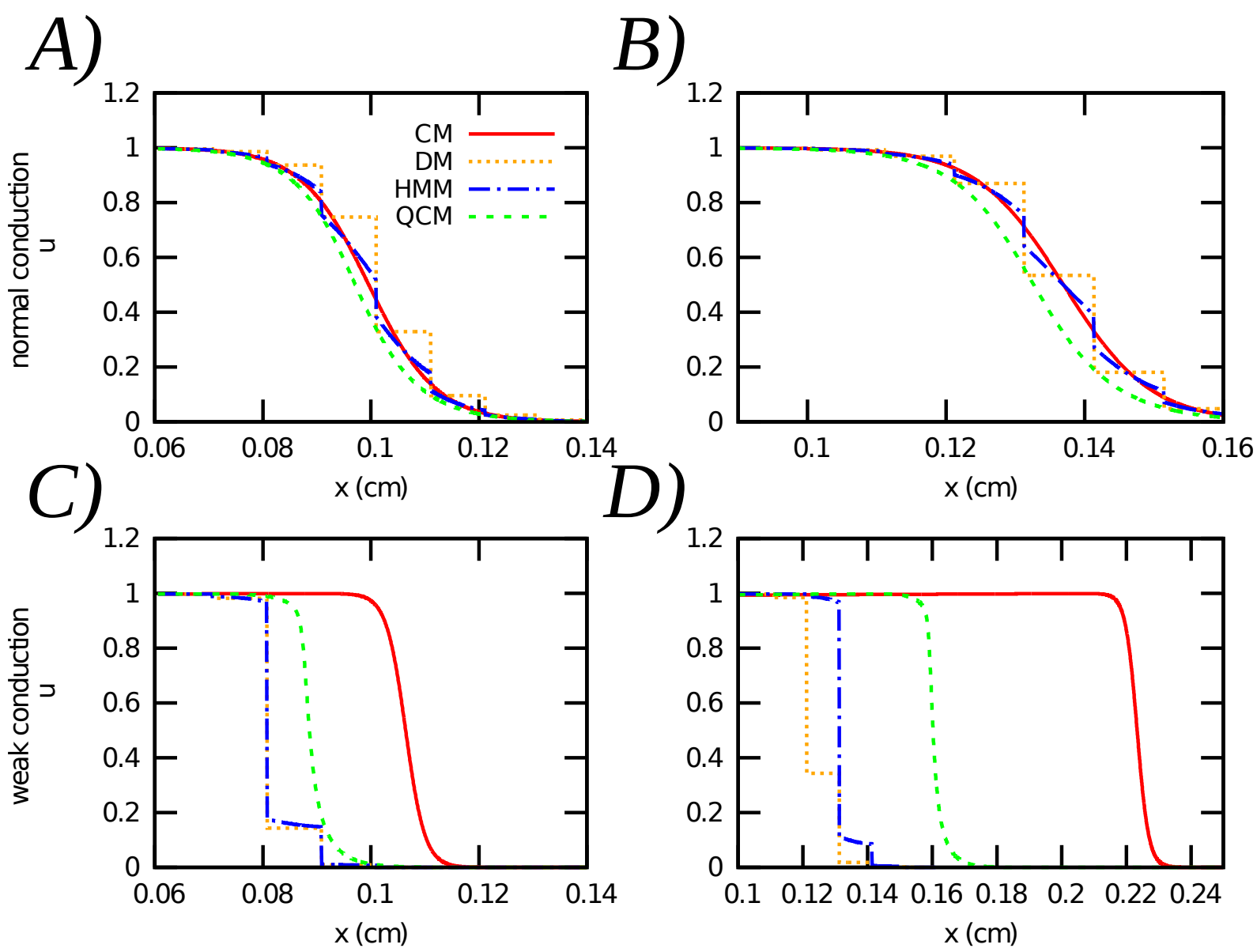

FIG. 2. Comparison of the spatial profiles of $u$ among the four models discussed in Sec.II A for two different conditions: normal conduction at $t=0.5 \mathrm{~ms}(\mathrm{~A})$ and $t=1.1 \mathrm{~ms}(\mathrm{~B})$; and weak conduction at $t=2.2 \mathrm{~ms}(\mathrm{C})$ and $t=8.3 \mathrm{~ms}(\mathrm{D})$. Both $Q C M$, $C M$ and $H M M$ were solved using a fine discretization. The parameter values employed in all simulations are: $a=0.2, b=0.5$, $k=36.0, \varepsilon=4.5 \cdot 10^{-5}, \bar{\theta}=\overline{\mathcal{X}}=1, \delta=0$. For the panels $(\mathrm{A})(\mathrm{B}), \bar{\sigma}=6.9 \times 10^{-4} \mu \mathrm{m}^{2} / \mathrm{ms}$. For the panels $(\mathrm{C}),(\mathrm{D})$ $\bar{\sigma}=5.6 \times 10^{-5} \mu m^{2} / m s$.

\section{B. Discretization-dependent homogenized model}

Here, we present an alternative homogenized model that tries to link the continuum and discrete approaches. The main idea of our model is to use also some information of the mesh or discretization, $\mathfrak{M}$. Here, we will use the value of $h$, which is the spatial discretization used by the numerical methods. Therefore, the coefficients of the PDEs depend on both $\mathfrak{L}$ and $\mathfrak{M}$, e.g., we have coefficients of the PDE that depend on $\ell$ and $h$. Therefore, we call our new model a Discretization Dependent Model $(D D M)$.

To compute the discrete Laplacian at position $x$ we need the evaluations of $u(x+\ell)$ and $u(x-\ell)$. However, after a continuum model or classical PDE is discretized we may have only access to $u(x+h)$ and $u(x-h)$, if we consider a homogeneous mesh with spatial discretization equals to $h$. Therefore, to obtain an approximation of the discrete Laplacian we look for an additive correction term $Q_{ \pm}$that satisfies:

$$
u(x \pm \ell)=Q_{ \pm}+u(x \pm h) .
$$

We obtain the function $Q_{+}$by the comparing the Taylor's expansion of $u(x+h)$ and $u(x+\ell)$, and equivalently for the function $Q_{-}$:

$$
\begin{aligned}
Q_{ \pm} & = \pm(\ell-h) \partial u+\left(\frac{\ell^{2}-h^{2}}{2}\right) \partial^{2} u \pm\left(\frac{\ell^{3}-h^{3}}{3 !}\right) \partial^{3} u \\
& +\left(\frac{\ell^{4}-h^{4}}{4 !}\right) \partial^{4} u \ldots
\end{aligned}
$$

Knowing $Q_{ \pm}$, we can use eq.(15) and eq.(16) to rewrite the discrete Laplacian:

$$
\partial_{\ell}^{2} u=\frac{u(x+h)+Q_{+}+u(x-h)+Q_{-}-2 u(x)}{\ell^{2}} .
$$

Rearranging terms and truncating the series we have:

$$
\partial_{\ell}^{2} u \approx \partial^{2} u+\left(\frac{\ell^{4}-h^{4}}{12 \ell^{2}}\right) \partial^{4} u(x) .
$$


Finally, we rewrite the series using Padé approximation $[21,25]$ to obtain:

$$
\partial_{\ell}^{2} u \approx \frac{\partial^{2}}{1-\left(\frac{\ell^{4}-h^{4}}{12 \ell^{2}}\right) \partial^{2}} u
$$

The reaction-diffusion equations casted as a discretization-dependent model are:

$$
\begin{aligned}
& \overline{\mathcal{X}} \partial_{t} u=\bar{\theta} f(u, v)+\left(\frac{\bar{\sigma}}{1-\kappa_{2} \partial_{x}^{2}}\right) \partial_{x}^{2} u \\
& \overline{\mathcal{X}} \partial_{t} v=\bar{\theta} g(u, v)+\left(\frac{\delta \bar{\sigma}}{1-\kappa_{2} \partial_{x}^{2}}\right) \partial_{x}^{2} v
\end{aligned}
$$

with $\kappa_{2}=\frac{\ell^{4}-h^{4}}{12 \ell^{2}}$, where $\ell^{2}$ is the length employed in the $D M$ and $Q C M$, and $h$ the mesh discretization.

The model $D D M$ has some very important properties:

- $D D M=Q C M$ when $h \rightarrow 0$. Taking $h \rightarrow 0$, eqs. $(20,21)$ are simplified to those of the quasicontinuum model $Q C M$, since $\kappa_{2} \rightarrow \kappa_{1}$.

- $D D M=C M$ when $\ell, h \rightarrow 0$. From the above we know that $D D M=Q C M$ when $h \rightarrow 0$. In addition, when $\ell \rightarrow 0$ eqs. $(13,14)$ are simplified to those of the homogenized continuum model $C M$. This is expected, since by $\ell \rightarrow 0$ we mean the discrete nature of the phenomena is not relevant and the classical continuum hypothesis is valid.

- $D D M=D M$ when $h \rightarrow \ell$. When the discretization mesh coincides with the underlying discrete system $D D M$ equations correspond to those of the discrete model $D M$.

Therefore, all the previous presented homogenized models, $C M, D M$ and $Q C M$ can be taken as particular cases of the new Discretization Dependent Model, DDM.

For more details on the numerical discretization of the models for both cases of uniform and non-uniform meshes see Appendix A.

\section{DISCRETE BIOCHEMICAL SYSTEMS}

In the previous section we have presented five approaches to model heterogeneous reaction-diffusion systems. Next, we choose three representative biochemical examples of heterogeneous reaction and diffusion to apply the different models.

We restrict our study to excitable systems composed of two phases, each one with different values for the reaction and diffusion coefficients. Each system alternates between an active region, $\mathcal{A}$ of length $l_{0}$ (gray colored area), and a passive one, $\mathcal{P}$ of length $l_{1}$ (white colored area), see Fig. 3. We study three biochemical systems with different underlying structural organization: the propagation of the action potential through cardiac tissue,
TABLE I. Table of values used in Eqs.(7-8) to model the three biochemical systems described in section III

\begin{tabular}{|c|c|c|c|}
\hline Parameters & Cardiac & Neuron & Chemical Reaction \\
\hline$k\left(\mathrm{~ms}^{-1}\right)$ & 36.0 & 800.0 & $80.0 \times 10^{-3}$ \\
\hline$\varepsilon$ & $4.5 \times 10^{-5}$ & $2.4 \times 10^{-4}$ & $1.1 \times 10^{-3}$ \\
\hline$\sigma_{0}\left(\mathrm{~cm}^{2} / \mathrm{ms}\right)$ & $1.035 \times 10^{-3}$ & $3.0 \times 10^{-6}$ & $3.2 \times 10^{-9}$ \\
\hline$\sigma_{1}\left(\mathrm{~cm}^{2} / \mathrm{ms}\right)$ & $1.035 \times 10^{-5}$ & $3.0 \times 10^{-3}$ & $1.6 \times 10^{-11}$ \\
\hline$\ell_{0}(\mu \mathrm{m})$ & 100 & 2.0 & 5.0 \\
\hline$\ell_{1}(\mu \mathrm{m})$ & 0.5 & 200 & 0.1 \\
\hline$\tau(\mathrm{ms})$ & 308 & 2.7 & 6300 \\
\hline$\lambda(\mathrm{cm})$ & 20 & 5.1 & 0.2 \\
\hline Speed $(\mathrm{cm} / \mathrm{ms})$ & $6.7 \times 10^{-2}$ & 1.9 & $2.3 \times 10^{-6}$ \\
\hline$\overline{\mathcal{X}}$ & 1 & $9.9 \times 10^{-3}$ & 1 \\
\hline $\bar{\theta}$ & $9.9 \times 10^{-1}$ & $9.9 \times 10^{-3}$ & $9.8 \times 10^{-1}$ \\
\hline$\delta$ & 0 & 0 & 1 \\
\hline
\end{tabular}

see Fig. 3(A), the propagation of the action potential through myelinated axons of neurons, see Fig. 3(B), and the Belousov-Zhabotinsky chemical reaction in aqueous droplets surrounded by oil, see Fig. 3(C).

These phenomena are modeled by the same general reaction-diffusion equations, as presented by the equations of the $H M M$ model, eqs. $(7,8)$. The space dependent functions are defined as follows: when $x \in \mathcal{A}$ we have $\theta=1, \mathcal{X}=\mathcal{X}_{0}$ and $\sigma=\sigma_{0}$; whereas when $x \in \mathcal{P}$ we have $\theta=0, \mathcal{X}=\mathcal{X}_{1}$ and $\sigma=\sigma_{1}$. In addition, the reaction terms are modeled by eqs. $(5,6)$ :

$$
\begin{aligned}
& \mathcal{X} \partial_{t} u=\theta k(u(1-u)(u-a)-u v)+\partial_{x}\left(\sigma \partial_{x} u\right), \\
& \mathcal{X} \partial_{t} v=\theta k(\epsilon(b u-v))+\delta \partial_{x}\left(\sigma \partial_{x} v\right) .
\end{aligned}
$$

All the parameters of the three biochemical models were carefully adjusted to obtain the distinct and characteristic wave propagation speeds and wave-lengths as reported in the literature, see Table I. In particular, we have chosen these three examples as they present very different temporal and spatial scales. Fig. 4 presents how the solutions depend on time and space for each of the three models. The neuron action potential is the fastest and the chemical reaction in droplets is the slowest system, see Fig. 4(A); in terms of spatial profile, the cardiac action potential has the longest wave-length and the chemical reaction in droplets the smallest one, see Fig. 4(B).

For the homogenized models, $C M, Q C M$ and $D D M$, the effective coefficients $\bar{\sigma}, \bar{\theta}, \overline{\mathcal{X}}$ are computed as follows: $\bar{\sigma}=\frac{\ell}{\ell_{0} / \sigma_{0}+\ell_{1} / \sigma_{1}}, \bar{\theta}=\ell_{0} / \ell, \overline{\mathcal{X}}=\ell_{0} / \ell$ for the neuron case, for cardiac and chemical reaction was $\overline{\mathcal{X}}=1$, where $\ell=\ell_{0}+\ell_{1}$. The other parameters are presented in Table I. 
A)

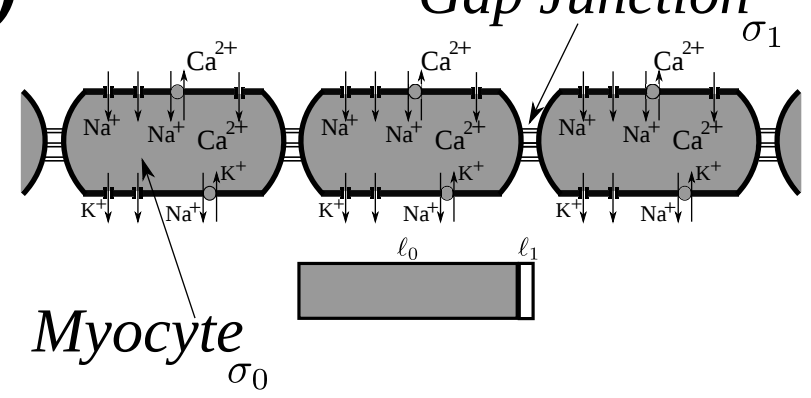

the effective conductivity of gap junctions decrease, $\sigma_{1}$, and can induce Conduction Block, and different types of arrhythmia [33, 34].

In these conditions, for reduced gap junction conductivity the $D M$ was shown to reproduce these features better than the $C M$, see [3].

We employ the modified FitzHugh-Nagumo model, see eqs. $(22,23)$, where the field $u$ corresponds to a renormalized membrane action potential and $v$ to a generic gate variable. Here, $\theta=1$ in the myocyte, whereas in the gap junctions we have only diffusion and $\theta=0$. See Table I for the other parameters of the model.

\section{B. Myelinated nerve axons}

Membrane action potential propagates along the axons of the neurons [35]. The accumulation of myelin along large regions of the axons induces a fast propagation of the impulse between the Ranvier nodes, short nodes where the electric impulse is intensified again [36], see Fig.3(B).

Therefore, this system is heterogeneous and multiscale, intercalating short nodes of Ranvier $\left(\sigma_{0}, \ell_{0}\right)$ and long Myelin Sheath $\left(\sigma_{1}, \ell_{1}\right)[3]$. We employ the modified FitzHugh-Nagumo model, see eqs. $(22,23)$, where the field $u$ corresponds to a renormalized membrane action potential and $v$ to a generic gate variable. Table I presents all the parameters of this model.

The lost of the thickness of the myelin sheath reduces the speed of propagation and may induce Conduction Block [3] and disturb the action potential propagation. Such alteration of the normal thickness of myelin is known to be related with multiple sclerosis [37].

FIG. 3. Sketch of the three discrete biochemical systems: Action potential propagation along active cardiac cells and passive gap junctions (A), action potential propagation along passive axons with myelin with active Ranvier nodes (B), and reaction front propagation in chemical active droplets and passive oil phase (C). Gray shaded areas of length $\ell_{0}$ and $\sigma_{0}$ correspond to active regions whereas white areas of length $\ell_{1}$ and $\sigma_{1}$ correspond to passive regions.

\section{A. Tissue of cardiac cells}

Membrane action potential in cardiac tissue propagates among individual cardiac cells, i.e. myocytes, by gap junctions. The propagation of the action potential is much faster inside the individual cells than through the gap junctions and therefore at this microscopic scale the tissue is heterogeneous, where the conduction depends on space $\sigma(x)$ and the tissue combines the scale of the myocytes $\ell_{0}=100 \mu \mathrm{m}$ with the size of the gap junctions $\ell_{1}=0.5 \mu \mathrm{m}$, see Fig.3 (A). Typically, under normal propagation the continuum approach is employed giving very good agreement with experimental data. However, under different cardiac diseases, such as infarct and ischemia,

\section{Chemical droplets in oil}

The Belousov-Zhabotinsky reaction is a classical chemical example of excitable media [7]. Excitation pulses and spiral waves fully develop in extended versions of this chemical reaction [38] in aqueous conditions. By the use of emulsions of water, oil and surfactant the activity of the reaction can be confined in small reactors formed by water droplets surrounded by a thin surfactant membrane immersed in the oil phase, see Fig.3(C). The size of the droplets can change from some nanometers [39] to the scale of hundred of microns [40]. Although the oil phase is passive and avoids any reaction, it permits the diffusion of the non-polar particles. One can model such excitable system by the use of activator-inhibitor system where two concentrations diffuse, and therefore we employ the same modified FitzHugh-Nagumo model, see eqs. $(22,23)$, with renormalized concentration in both fields $u$ and $v$, and with diffusion taking place in both equations $(\delta=1)$, see Table I. 


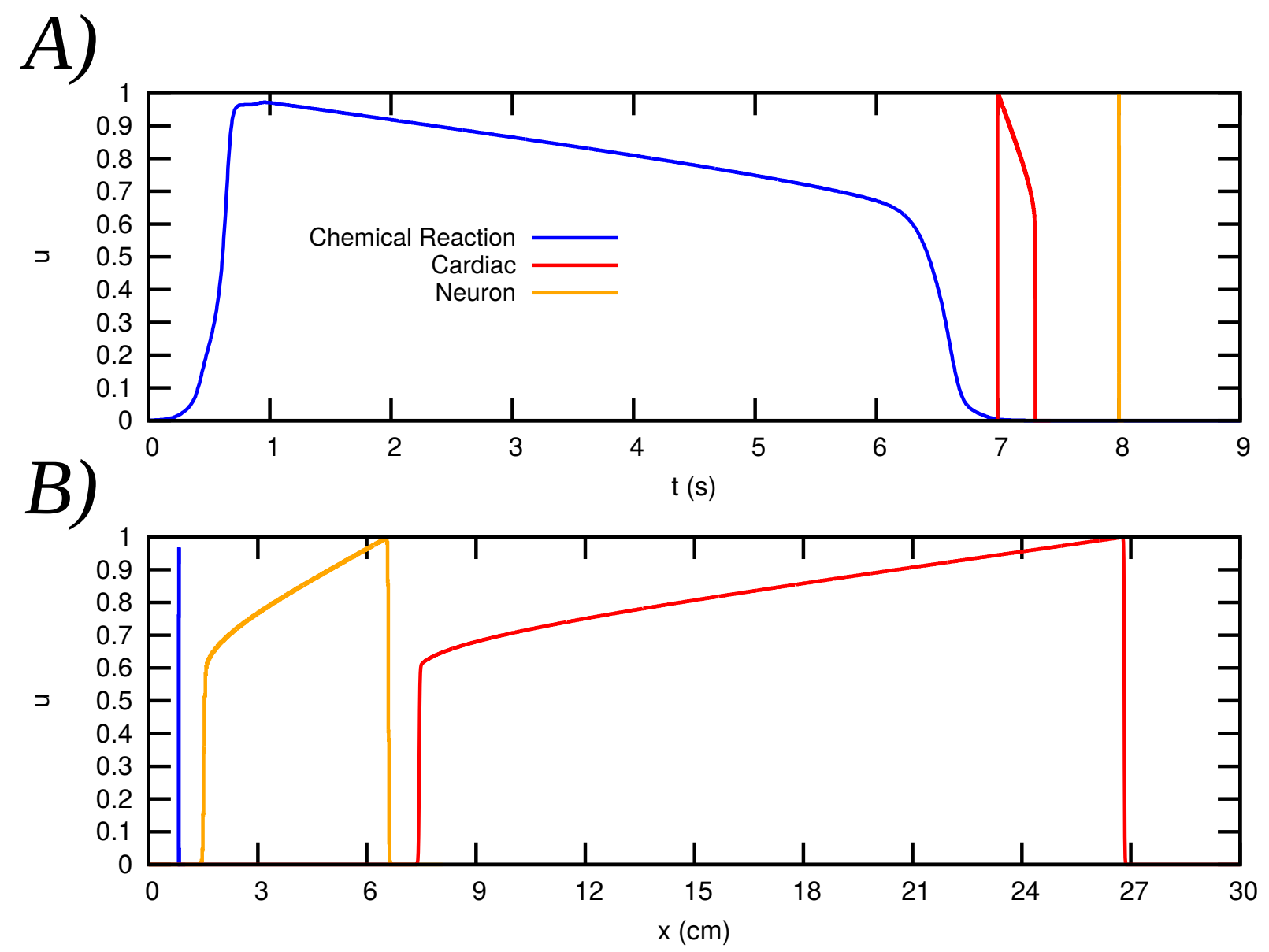

FIG. 4. Comparison of the local temporal evolution $u(t)(\mathrm{A})$ and the spatial profile $u(x)(\mathrm{B})$ for the three discrete biochemical reaction-diffusion systems, using the eqs. $(22,23)$ adapted for each case with parameter values from Table I.

\section{RESULTS}

We consider excitable waves in one-dimensional media under weak conductivity between consecutive elements. We study, for the three systems, propagation failure, i.e. conduction block, and the dependence of the total activation time on the distance to the location of the initial stimulus. In addition, we measure the dependence of the speed of the waves along the heterogeneous systems on the parameter $\sigma$, i.e. the conductivity or diffusion, and we evaluate the influence of the numerical discretization $h$ on the solutions for the different models. For all the three cases we obtained similar results: the $D M$ is a better approach than the continuous model $C M$ because it reproduces better the results of the $H M M$. The $D D M$ produces also similar speed of the waves as compared to the $H M M$, and is a good approach even for different values of the numerical discretization, $h$. The $D D M$ reproduces a similar speed of the heterogeneous model until the numerical scheme becomes unstable, which happens for $h>1.8 \ell$ as deduced before in [21].

\section{A. Tissue of cardiac cells}

The study of the speed of propagation of cardiac action potential is presented in Fig.5. For different values of $\bar{\sigma}$, Fig.5(A) presents the propagation speed obtained by the Heterogeneous Model HMM and the homogenized Continuous Model $C M$ with a fine discretization of $0.5 \mu \mathrm{m}$; and by the Discrete Model $D M$ with $\ell=100.5 \mu \mathrm{m}$. While the $C M$ does not give rise to conduction block, both $D M$ and $H M M$ produce conduction block for a similar value of the parameter $\bar{\sigma}$.

In Fig.5(B) we fix the discretization to $160 \mu \mathrm{m}$ for the homogenized $C M, Q C M$ and $D D M$ when varying the conductivity $\bar{\sigma}$ and compare the results with those of the $H M M$ with fine discretization. While $C M$ and $Q C M$ give rise to waves with small velocities and premature artificial conduction block, the $D D M$ shows better accuracy in the calculation of the wave velocity and in the determination of the conductivity where conduction block occurs, in this case at $\bar{\sigma}=4.2 \times 10^{-5} \mu^{2} / \mathrm{ms}$.

In Fig.5(C) we fix $\bar{\sigma}$, which produces a speed of 10 $\mathrm{cm} / \mathrm{s}$ with the model $H M M$, and compare the homogenized $C M, D D M$ and $Q C M$ models for different values 


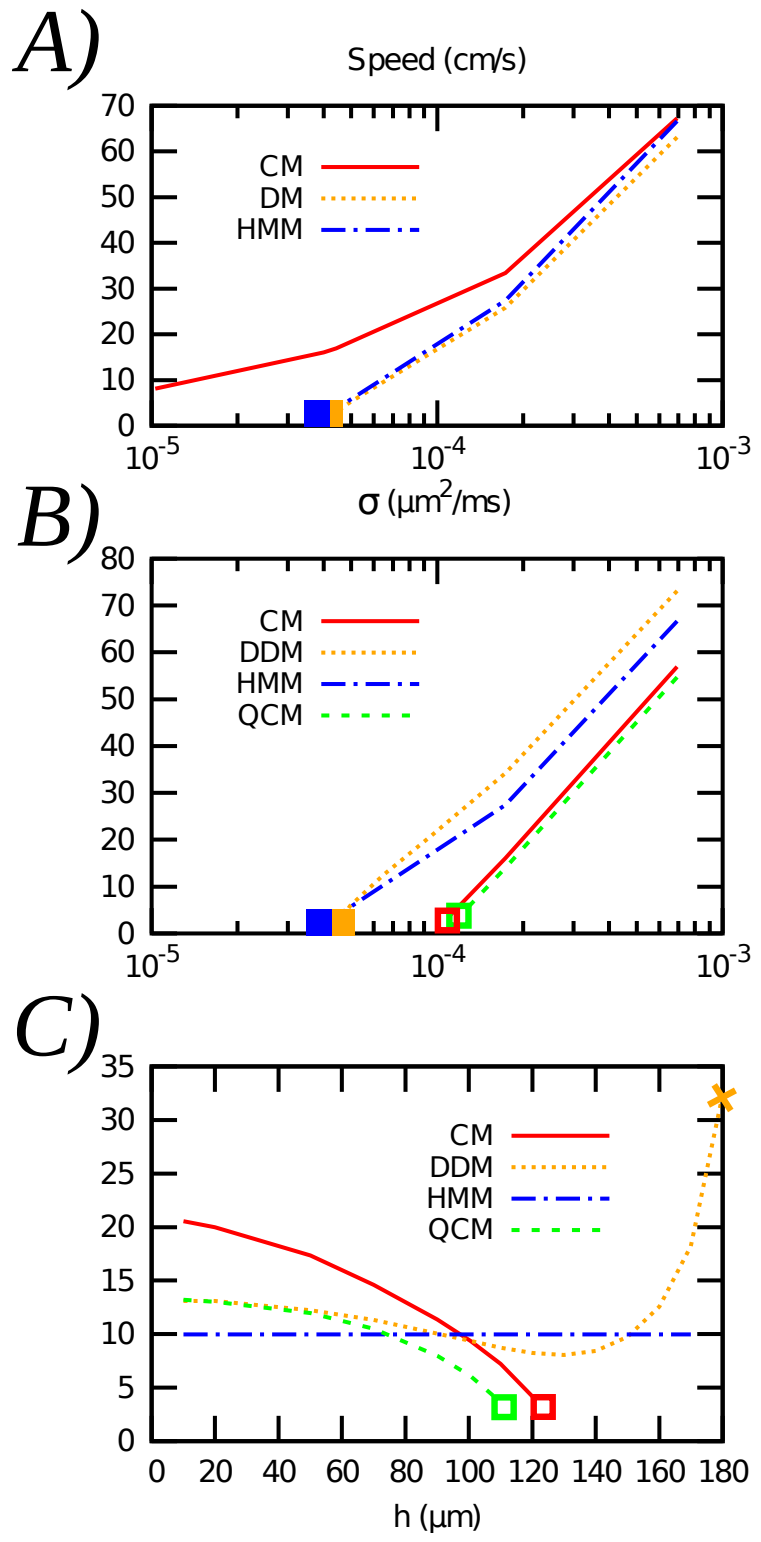

FIG. 5. Speed of propagation of cardiac action potential as function of the conductivity $\bar{\sigma}$ for (A) small discretization value ( $h=0.5 \mu \mathrm{m}$ for CM and HMM, and $h=100.5 \mu \mathrm{m}$ for DM); and (B) large discretization value $(h=160 \mu \mathrm{m})$ for CM, QCM and DDM. (C) Speed as a function of the discretization $h$ keeping constant $\bar{\sigma}$ at a weak conduction $6.5 \times$ $10^{-5}$. Conduction block and numerical instability are shown by squares and cross, respectively.

of discretization, $h$. For small discretization, $h \rightarrow 0$, the speed obtained with the $C M$ is artificially high in comparison with the $H M M$ model. However, the speed obtained with the $Q C M$ tends to the one measured in the $D D M$, which is relatively close to the speed of the $H M M$. The $C M$ and $Q C M$ models should not be used with a discretization larger than $120 \mu \mathrm{m}$, since the results suggest a conduction block that does not occur for this values of $\bar{\sigma}$. The $D D M$ model allows the use of larger values of discretization than the others, and only stops near $180 \mu \mathrm{m}$ due to numerical instabilities, denoted by a cross in the figure. With a large range for $h$, between $10 \mu m$ and 160 $\mu m$, the $D D M$ reproduces very well the velocity obtained with the $H M M$. The numerical instability occurs because we use a semi-implicit method, as previously described in [21] and its supplementary material.

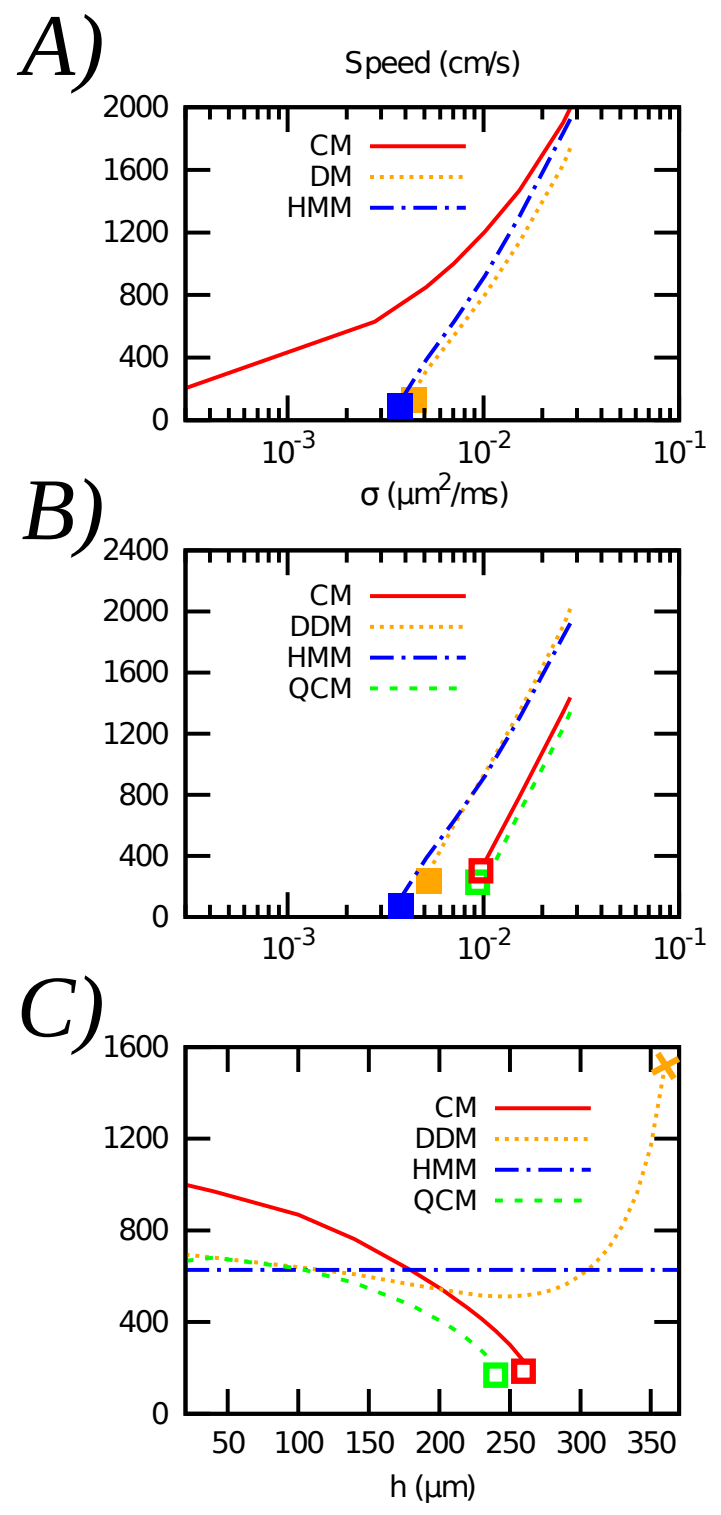

FIG. 6. Speed of propagation of action potential along a myelinated nerve axon as function of the conductivity $\bar{\sigma}$ for (A) small discretization value ( $h=1 \mu \mathrm{m}$ for CM and HMM, and $h=202 \mu \mathrm{m}$ for $\mathrm{DM}$ ); and (B) large discretization value $(h=300 \mu m)$ for CM, QCM and DDM. (C) Speed as a function of the discretization $h$ keeping constant $\bar{\sigma}$ at a weak conduction $7.1 \times 10^{-3}$. Conduction block and numerical instability are shown by squares and cross, respectively. 


\section{B. Myelinated nerve axon}

The resulting speed of action potential propagation along a myelinated axon of a neuron under different conditions is shown in Fig.6. As mentioned before, both temporal and spatial scales of this phenomenon are very different from the previous one. Nevertheless, the performance of each tested models is very similar.

For small conductivity, waves cannot propagate and produce conduction block. This phenomenon is observed in Fig.6(A) for the Heterogeneous Model HMM and the homogenized $D M$. However, $C M$ with fine discretization $(1 \mu \mathrm{m})$ does not reproduce the conduction block.

We compare also the $H M M$ with the homogenized $C M, Q C M$ and $D D M$ when the conductivity $\bar{\sigma}$ is varied, keeping fix the discretization at $300 \mu \mathrm{m}$ in Fig.6(B). The DDM can reproduce with better accuracy than $C M$ and $Q C M$ the conduction block that occurs when $\bar{\sigma}=3.8 \times 10^{-3}$.

In Fig.6(C) we compare the model $H M M$ with the homogenized $C M, Q C M$ and $D D M$ with $\bar{\sigma}=7.1 \times 10^{-3}$, corresponding to a speed of $628.8 \mathrm{~cm} / \mathrm{s}$. For small discretization, $h \rightarrow 0$ the $C M$ is far away from $H M M$, whereas $Q C M$ and $D D M$ converge to the same value and relatively close to the speed obtained with the $H M M$. The $C M$ and $Q C M$ cannot be used with a discretization larger than $250 \mu m$ and $230 \mu m$, respectively. The $D D M$ allows the use of larger discretization and stops near 380 $\mu m$ due to numerical instability. Between $1 \mu m$ and 350 $\mu m$ our $D D M$ is very effective in reproducing the velocity of the $H M M$.

\section{Chemical droplets in oil}

Diffusion coupled to the nonlinear chemical reactions can produce the propagation of reaction fronts. The dependence of the velocity on the different parameters is shown in Fig.7.

For small diffusivity, chemical waves cannot propagate and give rise to propagation block, see Fig.7(A), for the Heterogeneous Model $H M M$ and the homogenized $D M$. However, $C M$ with a fine discretization, $h=0.1 \mu \mathrm{m}$, cannot reproduce the conduction block.

We compared also the $H M M$ with the homogenized $C M, Q C M$ and $D D M$ when the conductivity $\bar{\sigma}$ is varied, keeping fix the discretization of $7.5 \mu \mathrm{m}$ in Fig.7(B). The $D D M$ can reproduce the conduction block that occurs at $\bar{\sigma}=3.6 \times 10^{-7}$ with better accuracy than $C M$ and $Q C M$.

In Fig.7(C) we compare the model HMM with the homogenized $C M, Q C M$ and $D D M$ with $\bar{\sigma}=4.6 \times 10^{-7}$, which corresponds to a speed of $14.5 \mathrm{~m} / \mathrm{s}$. We vary the discretization from $1 \mu \mathrm{m}$ to $8 \mu \mathrm{m}$. For small discretization, $h \rightarrow 0$ the $C M$ is far away from $H M M$, whereas $Q C M$ and $D D M$ converge to the same value that is relatively close to the speed obtained by the $H M M$. The $C M$ and $Q C M$ cannot use discretizations larger than $5.5 \mu \mathrm{m}$ and $4.5 \mu \mathrm{m}$, respectively. The $D D M$ allows larger values

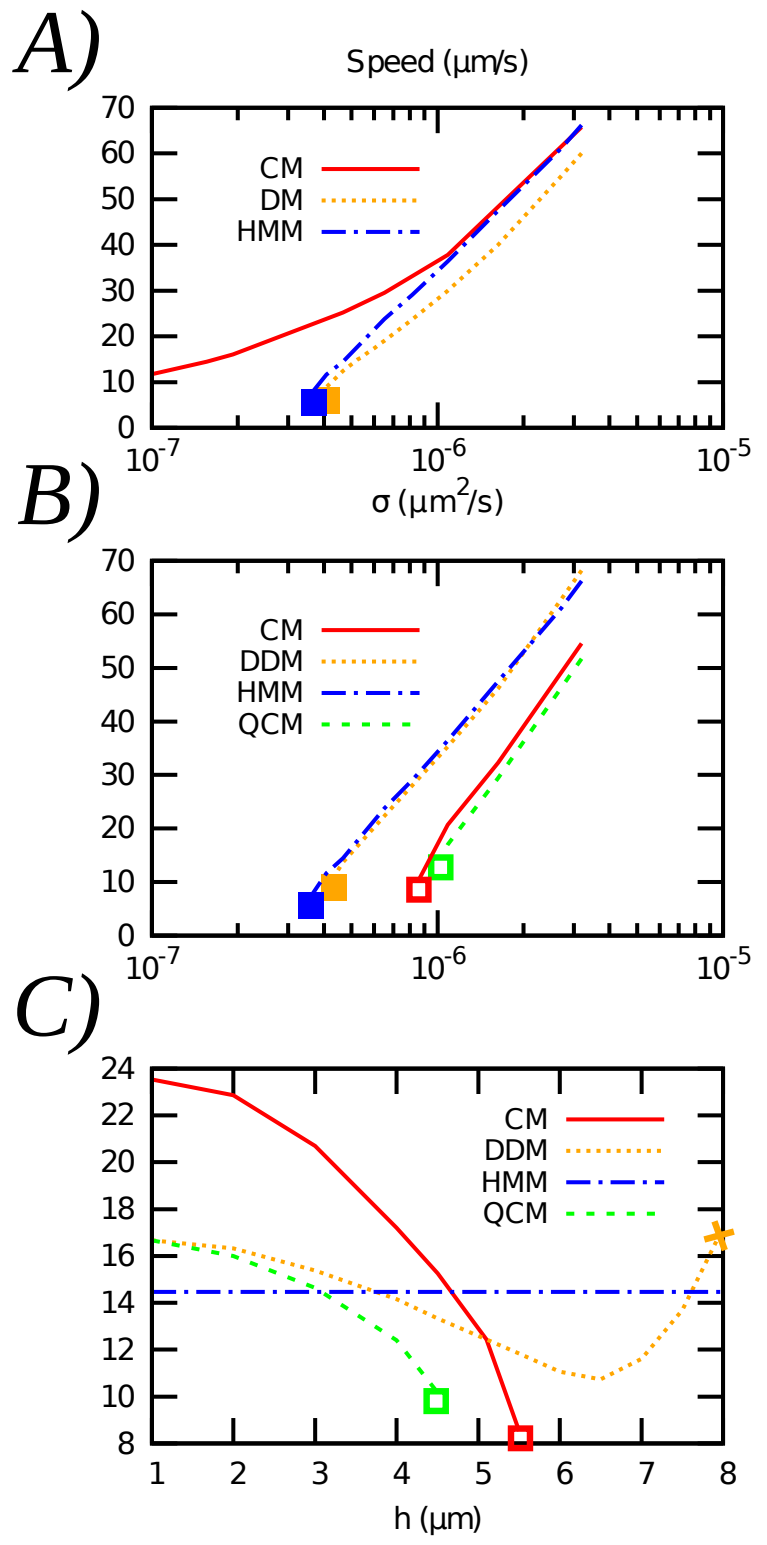

FIG. 7. Speed of wave propagation for the system with chemical droplets in oil as function of the diffusivity $\bar{\sigma}$ for (A) small discretization value $(h=0.1 \mu \mathrm{m}$ for $C M$ and $H M M$, and $h=5.1 \mu \mathrm{m}$ for $D M$ ); and (B) large discretization value $(h=7.5 \mu m)$ for CM, QCM and DDM. (C) Speed as a function of the discretization $h$ keeping constant $\bar{\sigma}$ at a weak diffusion $4.6 \times 10^{-7}$. Conduction block and numerical instability are shown by squares and cross, respectively.

of discretization to be used and stops near $8 \mu \mathrm{m}$ due to numerical instability. In the range of $1 \mu \mathrm{m}$ and $7.5 \mu \mathrm{m}$ our $D D M$ is very effective in reproducing the velocity of the $H M M$. 

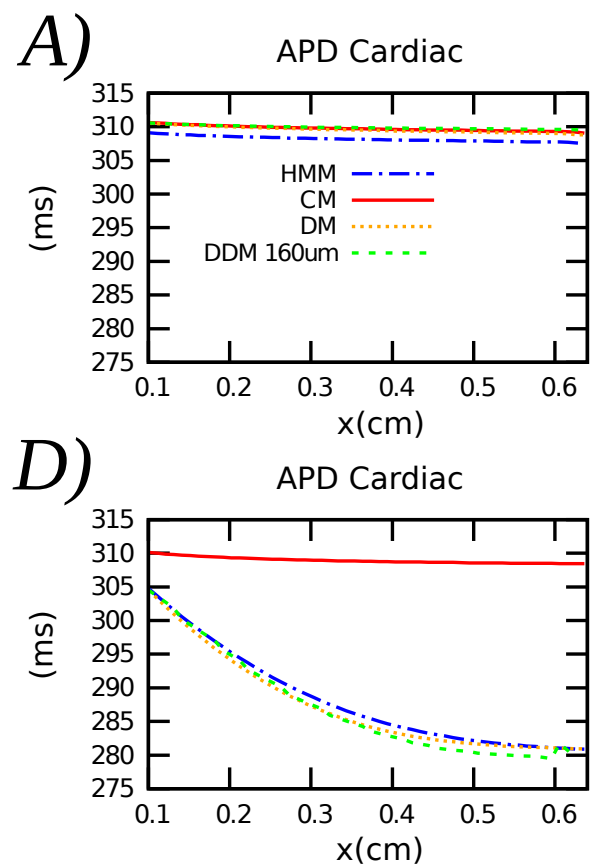

B)
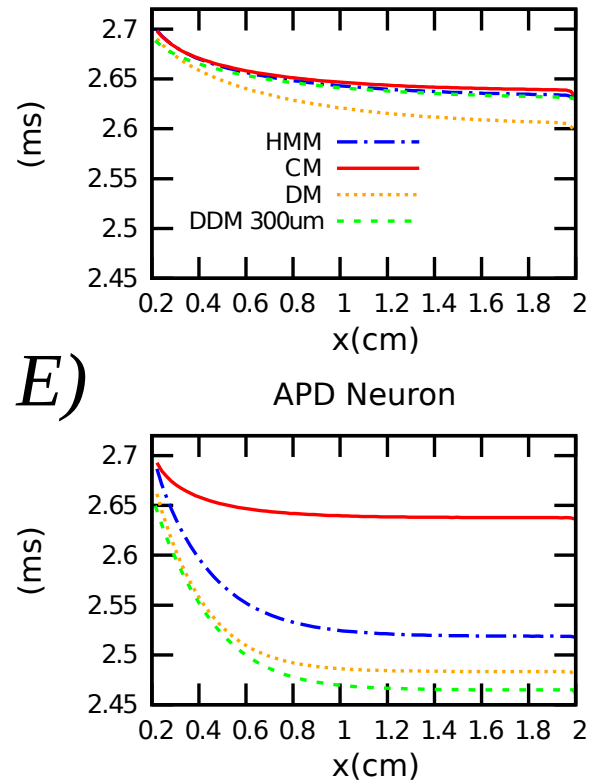

APD Neuron

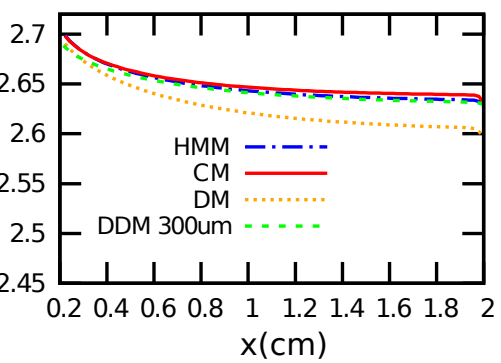

APD Neuron

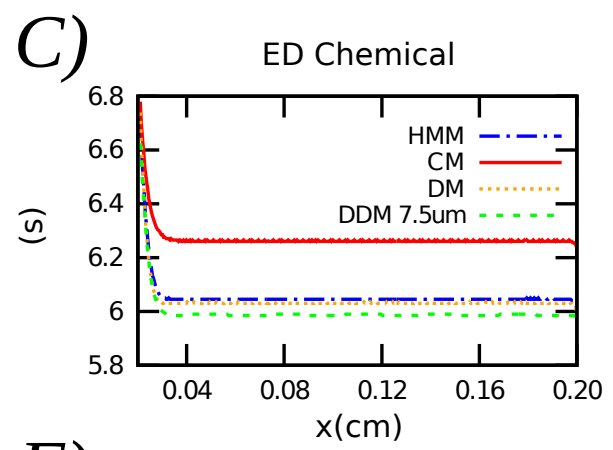

F)

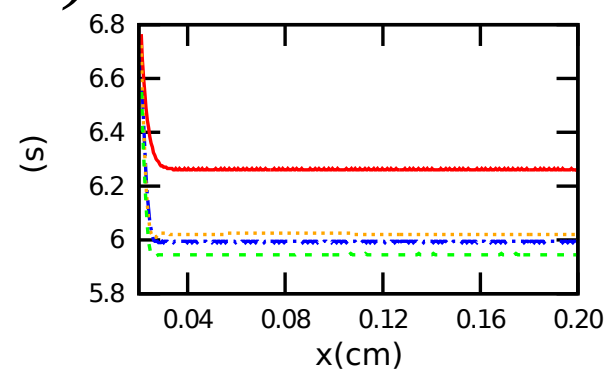

FIG. 8. Action Potential Duration (APD) and excitation duration (ED) dependence on the distance to the initial source. APD for the 64 cardiac cells for normal (A) and reduced conductivity $\left(\sigma=6.9 \times 10^{-4}\right)(\mathrm{D})$. APD for the myelinated axons with 100 myelin sheath for normal $(\mathrm{B})$ and reduced conductivity $\left(\sigma=7.0 \times 10^{-3}\right)(\mathrm{E})$. ED for the 400 chemical Droplets for normal $(\mathrm{C})$ and reduced diffusivity $\left(\sigma=6.5 \times 10^{-7}\right)(\mathrm{F})$.

\section{Transitory dynamics}

In the previous section the speed of the waves is calculated when the excitable wave takes a stationary shape and moves with constant velocity. However, before the wave adopts the final stationary shape, velocity and thickness of the wave changes depending on the initial condition. The local thickness of the excitable pulse, corresponding to action potential duration (APD) for cardiac cells and neurons and to excitation time for the chemical reactions, depends on the distance to the source where wave is initiated, see Fig. 8 for two different examples in each of the three biochemical systems.

Here, we observe the main differences among the three systems. While for normal conditions the action potential in the cardiac tissue rapidly relaxes to the stationary shape and all the models reproduce constant APD in the space, see Fig.8(A), the action potential in the axon continuously changes with the distance and the stationary shape is still not achieved, see Fig.8(B). The chemical droplets is an intermediate case where the transitory dynamics occurs along the first $0.03 \mathrm{~cm}$, see Fig.8(C).

The behavior of the chemical systems does not depend on the diffusivity $\sigma$, compare panel (C) and (F) in Fig.8. In both cases, Excitation durations (ED) are not correctly computed with the $C M$, whereas ED distributions obtained with $D M$ and $D D M$ are close to those of the $H M M$. In the other two cases, the change with the decrease of conductivity is more relevant. In the $C M$, ED distribution does not change substantially with the modification of the conductivity. However, with the $D M$ and $D D M$ the distribution changes and follows closely the one computed with the $H M M$.

\section{DISCUSSION}

We have presented a discretization dependent model for the integration of discrete biochemical systems which merges good properties of a discrete model (e.g. velocity dependence on conductivity, conduction block, dependence on the distance to the initial condition) with the applicability and cost of a homogenized continuum model (e.g. integration on unstructured meshes, analytic calculations).

As a final test of our argument we constructed an onedimensional mesh with random discretization, where we can integrate the continuum, quasi-continuum and our discretization dependent model and compare them with the standard heterogeneous $H M M$ model, see Fig.9. It is interesting to point out that the discrete model can not be solved on this unstructured mesh, as it requires the exact topology of the underlying discrete system. Using this non-uniform mesh the speed obtained with the $D D M$ and the corresponding conduction block are in good agreement with the results obtained by the $H M M$, see Fig.9(A). In summary, $D D M$ outperforms the classical continuum model, $C M$, the Quasi-continuum approaches to discreteness, $Q C M$, and it is computationally 

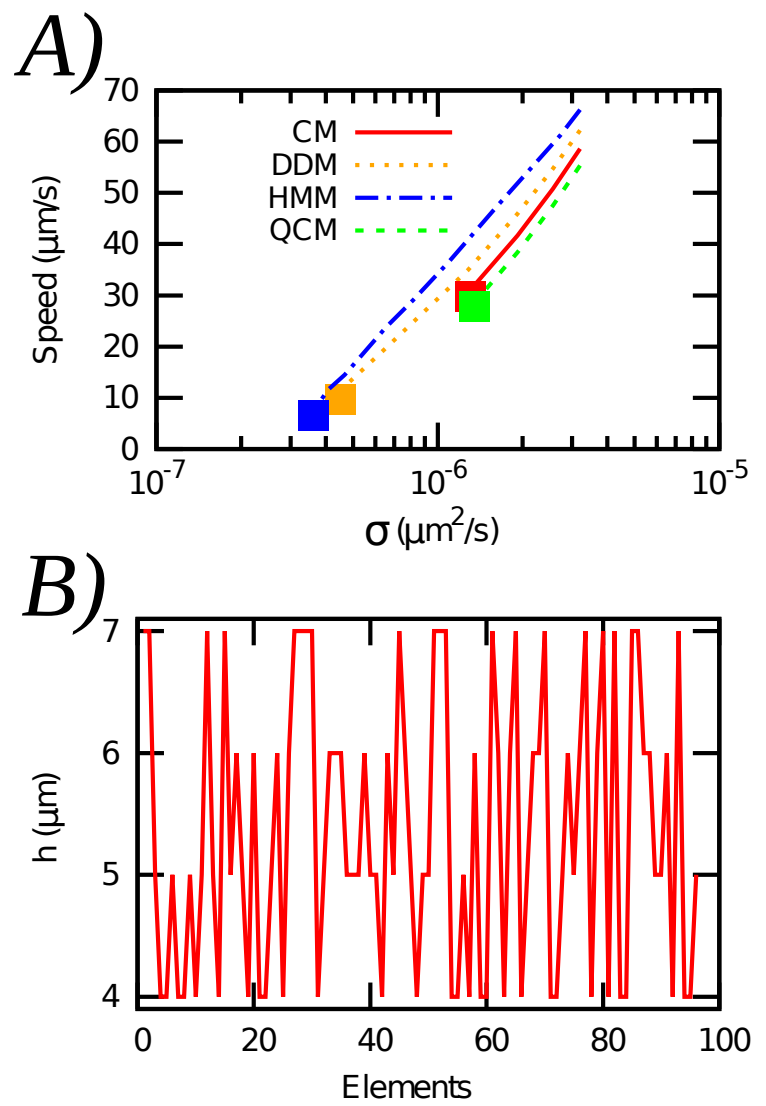

FIG. 9. (A) Speed of propagation of the concentration of the activator in the version of the model adapted to chemical droplets in oil as function of the diffusivity $\bar{\sigma}$ using (B) a non-uniform mesh with random discretization values between 4 and $7 \mu \mathrm{m}$ with an average of 5.35 and variance of 1.27 . Conduction block is shown by squares.

much cheaper than the heterogeneous model, HMM. As a matter of fact, $D D M$ was shown to be computationally cheaper than any other tested model in this work, including the $D M$, as it can be integrated with discretization values larger than those used by other models, see for instance Fig.5C.

It is interesting to note that both $D D M$ and $Q C M$ models mainly propose modifications on the diffusion term. This is similar to the modifications proposed in [41] to deal with cases of anomalous diffusion. Other techniques that have also been extensively used are nonlocal diffusion [42] and fractional diffusion. The $D M$ can be written as a non-local diffusion model. By taking two delta Dirac functions as the Kernel function of the integro-differential equation, we arrive at the discrete Laplacian in one-dimension. Therefore, our new model $D D M$ can also be seen as a modified non-local diffusion model. Non-local and fractional diffusion models can be equivalent, see [43]. Recently, fractional reactiondiffusion equations were used to model cardiac tissue [44] where all the information of the micro-structure of the tissue was projected to the fractional diffusion [45]. In particular, similar APD distributions studied here, see Fig. 8, can be found in [44]. Therefore, in the near future we will compare our new $D D M$ model to these other approaches: non-local, anomalous and fractional diffusion models. In this direction, our method can be also extended to more elaborated non-local couplings, to study the spread of pathological pulses in the brain [46].

For small discretization, the discretization-dependent model gives rise to a quasi-continuum model defined as a continuum approach to discreteness [23-25]. In turn, quasi-continuum models give rise to classical continuum model, when the inhomogeneity length $\ell$ can be neglected. Also, in the adequate limit, the discretization-dependent model reproduces the complete discrete model, which sometimes is a better description than continuum models. Therefore, all the previous presented homogenized models, $C M, D M$ and $Q C M$ can be taken as particular cases of the new Discretization Dependent Model, DDM.

We have compared the HMM with the homogenized versions of the continuous and discrete models. It is known that the homogenized versions of the continuous [11] and the discrete [10] models are good approaches to $H M M$ only for certain values of the parameters. If one of the conductivities is too small and the fraction close to the percolation limit, homogenization fails $[11,40]$. Under such conditions the excitation waves can break into pieces and produces irregular dynamics [17, 47, 48]. It may be interesting to extend the analysis here presented to two dimensional systems and compare the resulting dynamics.

Excitable waves can propagate in more complex topologies like networks $[13,49,50]$ where all the continuous models probably fail, and discrete models with complex interactions has to be consider. However, there are simpler networks like trees and some random networks where propagation failure has been also observed, where the $D D M$ may be also applied [51]. In addition, our model may be applied to study discrete breather, i.e. temporally periodic and strongly localized in space nonlinear structures, where the $Q C M$ was also applied with not completely satisfactory results $[52,53]$.

Finally, we would like to note that the proposed discretization-dependent models are not restricted to pattern formation in excitable media. They are rather general and can be used for other reaction-diffusion systems. There are multiple examples of patten formation in biology that are in the border of continuum and discrete descriptions, like the Turing mechanism [54], cell-to-cell communications [55] as well as other biological contexts at different spatial scales [56].

We acknowledge the support from CAPES, grant 88881.065002/2014-01 of the Brazilian program Science without borders, FAPEMIG, CNPq, UFJF, and from MINECO of Spain under the Ramon y Cajal program, grant number RYC-2012-11265. 


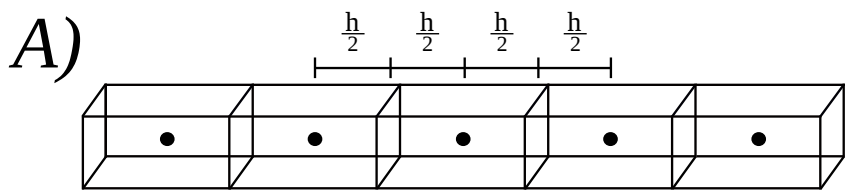

B)

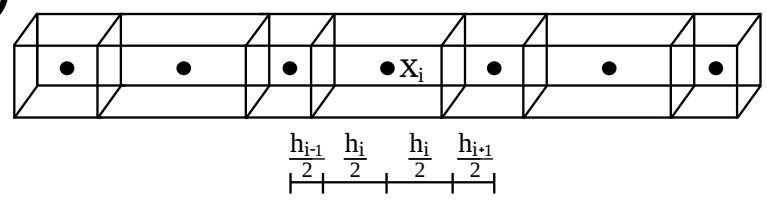

FIG. 10. Scheme of a uniform mesh A) with discretization $h$, and a non-uniform mesh B) with discretization $h_{i}$.

\section{Appendix A: Discretization of Models on Uniform and Non-uniform Meshes}

Here, we present the numerical schemes for the models for the cases of uniform and non-uniform meshes. We use operator splitting to separate the non-linear part that comes from the reactions and the linear term that comes from diffusion. More details of this scheme can be found in [21]. Here we focus on the discretization of the diffusion terms. For the classical $C M$ and $H M M$ models we have:

$$
\partial_{t} u=\partial(\sigma(x) \partial u)
$$

Here, $\sigma(x)$ varies on space either because we have the $H M M$ case or due to changes on the homogenized coefficient that may reflect non-periodic or nonregular information of the microscopic/discrete structure of the system, $\mathfrak{L}$. For example, cells may have different sizes and droplets alignment may be not regular so that the discrete characteristics may vary with space and the homogenized coefficient would reflect it: $\sigma(x)=\frac{\ell(x)}{\ell_{0}(x) / \sigma_{0}(x)+\ell_{1}(x) / \sigma_{1}(x)}$.

We assume also that our discretization may be nonuniform, i.e, $h$ varies. We can represent this either using a continuum approach, $h(x)$, or equivalently saying that our domain is discretized on $N$ volumes or elements, each with a size of $h_{i}$, with $i \in\{1, \cdots, N\}$. Fig. 10 presents examples of uniform and non-uniform meshes. By using the classical Finite Volume method to discretize space we have:

$\partial_{t} u_{i}=\frac{2}{h_{i}}\left[\sigma_{i+1 / 2}\left(\frac{u_{i+1}-u_{i}}{h_{i+1}+h_{i}}\right)-\sigma_{i-1 / 2}\left(\frac{u_{i}-u_{i-1}}{h_{i}+h_{i-1}}\right)\right]$,

where $u_{i}=u\left(x_{i}, t\right)$, with $x_{i}$ the coordinate of the center of the volume $i$ as shown in Fig. 10 .

In order to simplify the notation we define $T_{i \pm 1}^{\sigma}$ :

$$
T_{i \pm 1}^{\sigma}=\frac{2 \sigma_{i} \sigma_{i \pm 1}}{h_{i}\left(h_{i} \sigma_{i \pm 1}+h_{i \pm 1} \sigma_{i}\right)}
$$

where we have imposed the continuity of fluxes on faces $i+1 / 2$ and $i-1 / 2$ to arrive at this generalization of the harmonic mean.

Rewriting eq.(A2) we have:

$$
\partial_{t} u_{i}=\left[T_{i+1}^{\sigma}\left(u_{i+1}-u_{i}\right)-T_{i-1}^{\sigma}\left(u_{i}-u_{i-1}\right)\right] .
$$

To obtain a numerical solution we discretize time using Backward Euler. This generates a tridiagonal matrix that needs to be solved for each time step of the simulation.

For the special case of uniform mesh and constant $\sigma$ we find the classical second order approximation in space for the diffusion equation: $\partial_{t} u_{i}=\sigma \frac{u_{i+1}-2 u_{i}+u_{i-1}}{h^{2}}$. This is the discretization used to solve the homogeneous $C M$ on an uniform mesh or $D M$, replacing $h$ by $\ell$.

For a general non-uniform mesh, we cast $D D M$ basic equation as:

$$
\partial_{t} u=\left(\frac{1}{1-\kappa_{2} \partial^{2}}\right) \partial(\sigma(x) \partial u)
$$

Discretizing the numerator of the right hand side as before, and moving the denominator to the left hand side we have:

$\partial_{t} u_{i}-\kappa_{2 i} \partial^{2}\left(\partial_{t} u_{i}\right)=\left[T_{i+1}^{\sigma}\left(u_{i+1}-u_{i}\right)-T_{i-1}^{\sigma}\left(u_{i}-u_{i-1}\right)\right]$,

where $\kappa_{2 i}=\left(\ell_{i}^{4}-h_{i}^{4}\right) / 12 \ell_{i}^{2}$. Here, not only the mesh is non-uniform $\left(h_{i}\right)$, the discrete characteristics of the system may also vary with space, $\ell(x)$, with discretization also given by $\ell_{i}$.

Using the same spatial discretizaton on the left hand side we obtain:

$$
\begin{aligned}
\partial_{t} u_{i} & -\left[T_{i+1}^{\kappa_{2}}\left(\partial_{t} u_{i+1}-\partial_{t} u_{i}\right)-T_{i-1}^{\kappa_{2}}\left(\partial_{t} u_{i}-\partial_{t} u_{i-1}\right)\right] \\
& =\left[T_{i+1}^{\sigma}\left(u_{i+1}-u_{i}\right)-T_{i-1}^{\sigma}\left(u_{i}-u_{i-1}\right)\right],
\end{aligned}
$$

where

$$
T_{i \pm 1}^{\kappa_{2}}=\frac{2 \kappa_{2 i}}{h_{i}\left(h_{i}+h_{i \pm 1}\right)} .
$$

Finally, using Backward Euler for the time discretization we arrive at the following linear system that needs to be solved at each time step $k$ :

$$
\begin{aligned}
& -\left(T_{i+1}^{\kappa_{2}}+\Delta t T_{i+1}^{\sigma}\right) u_{i+1}^{n+1} \\
& +\left(T_{i+1}^{\kappa_{2}}+T_{i-1}^{\kappa_{2}}+1+\Delta t\left(T_{i+1}^{\sigma}+T_{i-1}^{\sigma}\right)\right) u_{i}^{n+1} \\
& -\left(T_{i-1}^{\kappa_{2}}+\Delta t T_{i-1}^{\sigma}\right) u_{i-1}^{n+1} \\
& =-\left(T_{i+1}^{\kappa_{2}}\right) u_{i+1}^{n}+\left(T_{i+1}^{\kappa_{2}}+T_{i-1}^{\kappa_{2}}+1\right) u_{i}^{n}-\left(T_{i-1}^{\kappa_{2}}\right) u_{i-1}^{n},
\end{aligned}
$$

where $u_{i}^{n}$ is the discretization of $u(x, t)=u\left(i h_{i}, n k\right)$, for the case of a uniform discretization of time, $k$. The numerical scheme (A9) is used to integrate the models $D D M$ 
and $Q C M$. For $Q C M$ we replace $\kappa_{2}$ by $\kappa_{1}$. Note that eq.(A9) also generates a tridiagonal matrix that needs to be solved for each time step of the simulation. Therefore, the computational cost of the different models, $D D M$, $Q C M, D M$ and $C M$, is the same and is mainly affected by the spatial discretization used, $h$.
[1] M. C. Cross and P. C. Hohenberg, Reviews of modern physics 65, 851 (1993).

[2] E. Meron, Physics reports 218, 1 (1992).

[3] J. Keener and J. Sneyd, Mathematical Physiology (Springer, 1998).

[4] J. D. Murray, Mathematical Biology (Springer-Verlag New York, 2002).

[5] S. Alonso, M. Bär, and B. Echebarria, Reports on Progress in Physics 79, 096601 (2016).

[6] J. G. Orlandi, J. Soriano, E. Alvarez-Lacalle, S. Teller, and J. Casademunt, Nature Physics 9, 582 (2013).

[7] F. Sagués and I. R. Epstein, Dalton transactions , 1201 (2003).

[8] R. Imbihl and G. Ertl, Chemical Reviews 95, 697 (1995).

[9] I. R. Epstein and V. K. Vanag, Chaos: An Interdisciplinary Journal of Nonlinear Science 15, 047510 (2005).

[10] S. Kirkpatrick, Reviews of modern physics 45, 574 (1973).

[11] S. Alonso, R. Kapral, and M. Bär, Physical review letters 102, 238302 (2009).

[12] D. A. G. Bruggeman, Annalen der Physik 416, 636 (1935).

[13] T. Isele and E. Schöll, New Journal of Physics 17, 023058 (2015).

[14] M. Spach and P. Dolber, Circ Res 58, 356 (1986).

[15] M. L. Hubbard, W. Ying, and C. S. Henriquez, Europace 9, vi20 (2007).

[16] J. Stinstra, C. Henriquez, and R. MacLeod, in Computers in Cardiology (2009) pp. 657-660.

[17] Y. Prudat and J. P. Kucera, Journal of Molecular and Cellular Cardiology 76, 46 (2014).

[18] B. G. de Barros, R. S. Oliveira, W. M. Jr., M. Lobosco, and R. W. dos Santos, Computational and Mathematical Methods in Medicine 2012 (2012), 10.1155/2012/824569.

[19] Y. Rudy and W. Quan, Circ Res 61, 815 (1987).

[20] I. V. Kazbanov, K. H. Ten Tusscher, and A. V. Panfilov, Scientific reports 6 (2016).

[21] C. M. Costa, P. A. A. Silva, and R. W. dos Santos, IEEE Transactions on Biomedical Engineering 63, 765 (2016).

[22] M. Toda, Progress of Theoretical Physics Supplement 59, 1 (1976).

[23] M. A. Collins, Chemical Physics Letters 77, 342 (1981).

[24] P. Rosenau, Physics Letters A 118, 222 (1986).

[25] P. Kevrekidis, I. Kevrekidis, A. Bishop, and E. Titi, Phys. Rev. E 65, 046613 (2002).

[26] I. Andrianov, J. Awrejcewicz, and D. Weichert, Mathematical Problems in Engineering 2010 (2009).

[27] L. Truskinovsky and A. Vainchtein, Continuum Mechanics and Thermodynamics 18, 1 (2006).

[28] N. Challamel, V. Picandet, B. Collet, T. Michelitsch, I. Elishakoff, and C. Wang, European Journal of Mechanics-A/Solids 53, 107 (2015).

[29] M. Charlotte and L. Truskinovsky, Journal of the Mechanics and Physics of Solids 60, 1508 (2012).

[30] J. M. Rogers and A. D. McCulloch, IEEE Transactions on Biomedical Engineering 41, 743 (1994).

[31] R. FitzHugh, Biophysical journal 1, 445 (1961).

[32] J. Nagumo, S. Arimoto, and S. Yoshizawa, Proceedings of the IRE 50, 2061 (1962).

[33] S. Rohr, J. P. Kucera, and A. G. Kléber, Circulation research 83, 781 (1998).

[34] J. P. Kucera, A. G. Kléber, and S. Rohr, Circulation Research 83, 795 (1998).

[35] B. P. Bean, Nature reviews. Neuroscience 8, 451 (2007).

[36] K.-A. Nave and H. B. Werner, Annual review of cell and developmental biology 30, 503 (2014).

[37] M. A. Friese, B. Schattling, and L. Fugger, Nature Reviews Neurology 10, 225 (2014).

[38] A. T. Winfree, Science 175, 634 (1972).

[39] V. K. Vanag and I. R. Epstein, Physical review letters 87, 228301 (2001).

[40] S. Thutupalli and S. Herminghaus, The European Physical Journal E 36, 91 (2013).

[41] L. G. Silva, D. C. Knupp, L. Bevilacqua, A. C. Galeão, and A. J. S. Neto, Computer Assisted Methods in Engineering and Science 21, 245 (2017).

[42] C. Bachmair and E. Schöll, The European Physical Journal B 87, 276 (2014).

[43] M. Delia and M. Gunzburger, Computers and Mathematics with Applications 66, 1245 (2013).

[44] A. Bueno-Orovio, D. Kay, V. Grau, B. Rodriguez, and K. Burrage, Journal of The Royal Society Interface 11, 20140352 (2014).

[45] A. Bueno-Orovio, I. Teh, J. E. Schneider, K. Burrage, and V. Grau, IEEE transactions on medical imaging 35, 2200 (2016).

[46] F. M. Schneider, E. Schöll, and M. A. Dahlem, Chaos: An Interdisciplinary Journal of Nonlinear Science 19, 015110 (2009).

[47] S. Alonso and M. Bär, Physical review letters 110, 158101 (2013).

[48] S. Alonso, R. W. dos Santos, and M. Br, PLOS ONE 11, 1 (2016).

[49] T. Isele, B. Hartung, P. Hövel, and E. Schöll, The European Physical Journal B 88, 104 (2015).

[50] J. G. Orlandi and J. Casademunt, Physical Review E 95, 052304 (2017).

[51] N. E. Kouvaris, T. Isele, A. S. Mikhailov, and E. Schöll, EPL (Europhysics Letters) 106, 68001 (2014).

[52] B.-F. Feng and T. Kawahara, Wave Motion 45, 68 (2007).

[53] C. Chong, M. A. Porter, P. Kevrekidis, and C. Daraio, Journal of Physics: Condensed Matter 29, 413003 (2017).

[54] L. Marcon and J. Sharpe, Current opinion in genetics \& development 22, 578 (2012).

[55] E. M. Rauch and M. M. Millonas, Journal of theoretical biology 226, 401 (2004).

[56] J. D. Murray, P. K. Maini, and R. T. Tranquillo, Physics Reports 171, 59 (1988). 